\title{
Impact of Contamination from Scattered Photons in Singles-Mode Transmission Data on Quantitative Small-Animal PET Imaging
}

\author{
Eric Vandervoort and Vesna Sossi \\ Department of Physics and Astronomy, University of British Columbia, Vancouver, British Columbia, Canada
}

\begin{abstract}
In previous work, we described and validated a method of scatter correction for singles-mode transmission data using experimental preinjection data acquired with a dedicated rodent PET scanner. In the current work, we investigated the impact that our method has on the quantitative accuracy of small-animal PET. Methods: This investigation had 3 stages. We first confirmed the general validity of our method by applying it to preinjection transmission data from a different imaging system (a larger dedicated primate scanner). For these data, we evaluated the accuracy of the reconstructed distributions of linear attenuation coefficients ( $\mu$-values). In the second stage, we applied our attenuationmap reconstruction and scatter correction procedure for postinjection transmission data acquired with the dedicated rodent scanner. For these studies, we investigated the quantitative accuracy of reconstructed emission images that use attenuation correction derived from postinjection transmission data. In the third stage, we compared our scatter correction method with 2 more commonly used alternatives (automated rescaling and segmentation of the attenuation-map images). Results: For the primate scanner data, the average reconstructed $\mu$-values with scatter correction were within 3\% of the expected values for water and soft tissue, whereas uncorrected values were $19 \%-26 \%$ lower than their expected values. For the postinjection transmission studies, we found that the correct average $\mu$-values and reconstructed activity concentrations consistent with wellcounter measurements were obtained only when scatter correction and emission contamination correction were applied to the transmission data. We also found that our transmission scatter correction provides more accurate $\mu$-values and better image quantification than either rescaling or segmentation. Conclusion: Using different imaging systems (primate and rodent) and different scanning protocols (before and after injection), we found that our transmission scatter correction is more accurate (for both reconstructed $\mu$-values and activity concentrations) than the existing alternatives.
\end{abstract}

Key Words: small-animal PET; attenuation correction; singlesmode transmission; postinjection transmission; quantitative PET

J Nucl Med 2008; 49:1852-1861

DOI: 10.2967/jnumed.108.051193

Received Jan. 29, 2008; revision accepted Aug. 1, 2008.

For correspondence please contact: Eric Vandervoort, Ottawa Hospital Cancer Centre, 501 Smyth Rd., Ottawa, ON K1H 8L6, Canada.

E-mail: evandervoort@toh.on.ca

COPYRIGHT $\odot 2008$ by the Society of Nuclear Medicine, Inc.
A ttenuation correction factors, which are necessary for quantitatively accurate PET, can be obtained using a singles-mode transmission scan. These scans are capable of a higher counting rate than are conventional coincidencemode transmission acquisitions. However, contamination from scattered photons and from emission activity (in postinjection transmissions) can be a significant problem. A correction for emission contamination can be obtained directly by acquiring a "mock" transmission scan (1), in which the transmission source is removed or shielded from the detectors. In previous work (2), we presented a scatter correction for singles-mode transmission data and validated our results using preinjection data from the microPET Focus 120 (Siemens Medical Solutions USA, Inc.) (3,4), a dedicated rodent scanner.

In this work, we applied our method of scatter correction using transmission data from the microPET Focus 220 (Siemens Medical Solutions USA, Inc.) (5), a scanner suitable for primate studies. The purpose of these studies was to test the general validity of our scatter correction for larger objects (subject to more photon attenuation and higher amounts of both single and multiple scatter) and for a larger-diameter PET scanner (with a decreased solid angle for the detection of both scattered and unscattered photons). Then, using data from the microPET Focus 120, we tested our analytic scatter correction for postinjection transmission data. For both scanners, we evaluated the accuracy of the reconstructed linear attenuation coefficients ( $\mu$-values). For the microPET Focus 120 data, we also investigated the quantitative accuracy of reconstructed emission images in terms of absolute quantitative units $(\mathrm{MBq})$. For the attenuation and scatter correction applied to the emission data, we used attenuation maps reconstructed with and without correction for scatter and emission contamination obtained from mock scans.

Singles-mode transmission data are not typically corrected for scatter. More commonly, these data are reconstructed and the resulting attenuation-map images are segmented. In these segmentation algorithms, each voxel of the attenuation map is classified as a particular type of tissue and then reassigned a 
theoretic $\mu$-value for that tissue type. To save time and reduce interuser variability, most segmentation routines are automated or require minimal user intervention $(6,7)$. Alternatively, the reconstructed attenuation-map data could simply be rescaled using the knowledge that most biologic materials (e.g., soft tissue and muscle) have attenuation coefficients close to that of water. Using 2 simple phantom configurations (water cylinders filled with uniform concentrations of ${ }^{18} \mathrm{~F}$ activity), we examined how these 2 alternatives to transmission scatter correction can lead to both qualitative and quantitative errors in the reconstructed activity concentrations for PET images.

\section{MATERIALS AND METHODS}

\section{Primate Scanner Studies}

The microPET Focus 220, a dedicated PET scanner for primates (5), uses LSO scintillator blocks each consisting of a $12 \times 12$ crystal array (crystal size, $1.5 \times 1.5 \times 10 \mathrm{~mm}$ ). Each block is coupled to a position-sensitive photomultiplier tube by a fiberoptic bundle. There are a total of 504 detectors per ring and 48 crystal rings. The ring diameter of the scanner is $261 \mathrm{~mm}$, and the axial length is $77 \mathrm{~mm}$. All transmission data for this scanner were acquired using a pointlike ${ }^{57} \mathrm{Co}(122-\mathrm{keV}$ photon emitter) transmission source that rotates and translates axially through the scanner field of view. For photons emitted by the ${ }^{57} \mathrm{Co}$ source, an extremely narrow energy window between 120 and $125 \mathrm{keV}$ is used to reduce the acquisition of scattered photons during transmission scanning.

Singles-mode transmission data were acquired for a $50-\mathrm{mm}$ radius water cylinder offset vertically from the center of the scanner field of view by $27 \mathrm{~mm}$. For this study, no activity was present in the cylinder, the blank data were acquired for $2 \mathrm{~h}$, and the duration of the transmission scan was $27 \mathrm{~min}$. The primate transmission data were acquired before injection (no emission contamination was present in the scanner) using a $6.5-\mathrm{kg}$ Macaca nemestrina monkey. Our scatter correction was originally validated for rats and mice (2), which typically weigh 300 and $30 \mathrm{~g}$, respectively. For this study, the blank and transmission data were acquired for $2 \mathrm{~h}$ and $45 \mathrm{~min}$, respectively. Because these scan durations were much longer than what is typically used for smallanimal PET transmission scans, we expected that these data would provide low-noise images in which the systematic effects of scatter would be more evident.

All transmission and blank data were processed using singleslice rebinning (8) with a maximum oblique angle of $8.9^{\circ}$ (corresponding to a maximum ring difference of 23). All microPET Focus 220 transmission data were reconstructed using ordered-subsets transmission (9), a maximum a posteriori reconstruction algorithm for transmission tomography. For all our reconstructions, the size of the reconstructed attenuation-map images was $256 \times 256$, and we used 20 iterations with 4 subsets, a smoothing intensity parameter $\beta=2^{8}$, and a Huber penalty function (10) with a cutoff parameter of $\delta=0.5 \times \mu_{\text {water }}$, where $\mu_{\text {water }}$ is the linear attenuation coefficient for water at the appropriate photon energy. The Huber penalty function applies heavier quadratic smoothing for neighboring voxels with reconstructed $\mu$-values that differ by less than $\delta$. For differences greater than $\delta$, linear smoothing is applied to discourage oversmoothing of abrupt changes, which should occur at the boundaries between different tissue types.

For reconstruction of the transmission data, we used our previously described scatter correction and reconstruction procedure (2). Our transmission scatter correction is similar to the single scatter simulation (11) that is now commonly used for coincidence-mode emission data. We use an estimate of the attenuation map (an image representing the distribution of linear attenuation coefficients within the subject being imaged) and the Klein-Nishina scattering crosssection to compute the contribution from single scattered (one Compton scatter interaction) photons to each crystal pair in the PET detector array during the transmission scan. Because our scatter correction requires an estimate of the attenuation map for its calculation, 5 distinct steps are needed:

1. We first reconstruct an initial attenuation map with no correction for scatter.

2. This uncorrected attenuation-map image is processed into a histogram of linear attenuation coefficients ( $\mu$-values). The image is then automatically rescaled so that the maximum histogram bin corresponds to the correct $\mu$-value for water.

3. We compute scatter sinogram data using the rescaled attenuation map.

4. Each scatter sinogram is rescaled to account for multiple scatter and scatter from outside the FOV and then subtracted from the experimental transmission data.

5. The scatter-corrected transmission data are then reconstructed.

\section{Studies Using Postinjection Transmission Data}

Experiments. Experimental postinjection transmission data were acquired using the microPET Focus 120. The microPET Focus 120 and microPET Focus 220 are similar scanners that share hardware and software. For example, they use the same block detector modules, have the same crystal size $(1.5 \times 1.5 \times 10$ $\mathrm{mm}$ ), have the same number of axial crystal rings (48), and share the same acquisition software. The primary difference between the 2 scanners is that the microPET Focus 120 has fewer crystals per axial ring (288 in total), arranged in a smaller-diameter $(140 \mathrm{~mm})$ ring. For each experiment using this scanner, 2 sets of singlesmode transmission data were acquired using pointlike ${ }^{68} \mathrm{Ge}$ (a positron emitter) and ${ }^{57} \mathrm{Co}$ (a $122-\mathrm{keV}$ photon emitter) transmission sources. The average measured energy resolution of the system is $18 \%$ at $511 \mathrm{keV}$ and $35 \%$ at $122 \mathrm{keV}$. For the annihilation photons emitted by the ${ }^{68} \mathrm{Ge}$ source, an energy discrimination window between 350 and $750 \mathrm{keV}$ is used. All transmission and blank data that were acquired using a ${ }^{68} \mathrm{Ge}$ source in this work were precorrected for contamination because of the naturally occurring ${ }^{176} \mathrm{Lu}$ radioactivity in the crystals (12). For the ${ }^{57} \mathrm{Co}$ source, the same energy discrimination settings were used as those described previously for the microPET Focus 220.

We acquired postinjection data for a 30-mm-radius water cylinder with a ${ }^{68} \mathrm{Ge}$ line source, containing approximately 1.3 $\mathrm{MBq}$, attached to the side. The length of the line source was 115 $\mathrm{mm}$, which is larger than the axial length of the scanner $(77 \mathrm{~mm})$; we therefore estimated that approximately $0.9 \mathrm{MBq}$ of the linesource activity was present inside the scanner during the emission scan. Two sets of transmission and blank scans were acquired using a $14-\mathrm{MBq}{ }^{68} \mathrm{Ge}$ source and a $100-\mathrm{MBq}{ }^{57} \mathrm{Co}$ source. The 
duration of all blank, transmission, and mock scans was 17 min. The duration of the emission scan was $30 \mathrm{~min}$.

We also acquired transmission and blank data for a healthy rodent injected with ${ }^{18} \mathrm{~F}$-FDG. We attached to the rat's shoulder a small glass vial (diameter of $10 \mathrm{~mm}$ and length of $34 \mathrm{~mm}$ ) filled with a solution of ${ }^{18} \mathrm{~F}(2.0 \mathrm{~mL})$. This vial was meant to approximate an external tumor similar to oncology studies in which human cancer cells are transplanted close to the surface of a rodent's skin $(13,14)$. At the beginning of the emission scan, we estimated that $6 \mathrm{MBq}$ of ${ }^{18} \mathrm{~F}$ activity were present in the simulated tumor and about $24 \mathrm{MBq}$ of ${ }^{18} \mathrm{~F}-\mathrm{FDG}$ activity were distributed throughout the rat. At the time of the rodent studies, the ${ }^{68} \mathrm{Ge}$ and ${ }^{57} \mathrm{Co}$ transmission sources had activities of 10 and $72 \mathrm{MBq}$, respectively. For this study, all blank and transmission scans were acquired for $17 \mathrm{~min}$, whereas the mock scans were acquired for only $8.5 \mathrm{~min}$. This duration is more typical in animal studies, in which the mock acquisitions are short to minimize total scan times. The duration of the emission scan was $20 \mathrm{~min}$.

Transmission Data Reconstruction. The postinjection transmission data acquired using the microPET Focus 120 were reconstructed using ordered-subsets transmission (9) with the same parameters as were described previously with the exception of image size, which was $128 \times 128$ for these data, and maximum oblique angle, which was $13.7^{\circ}$ (corresponding to a maximum ring difference of 23) for single-slice rebinning with this scanner. For this analysis, we compared reconstructions performed with 3 different combinations of emission-contamination correction and scatter correction:

1. No correction. For these data, we reconstruct with no correction for scatter or for emission contamination, to determine the combined importance of these effects.

2. Correction for emission contamination only. For this reconstruction, we subtract the experimentally measured mock scan sinograms (corrected for radioactive decay of the emission tracer) from the transmission data. We then reconstruct the data with no correction for transmission photon scatter.

3. Correction for both emission contamination and scatter. This correction also subtracts the decay-corrected mock scans from the transmission data but applies our scatter correction and reconstruction procedure.

Emission Data Reconstruction. The PET emission data, acquired using the microPET Focus 120, were sorted into 3-dimensional sinograms. The emission sinogram data were corrected for random events by subtracting data acquired in a delayed coincidence window. The sinogram data were then corrected for detector normalization using a component-based method (15). Using Fourier rebinning (16), the 3-dimensional sinograms were rebinned into a smaller set of 2-dimensional sinograms and then reconstructed using 2-dimensional filtered backprojection with a ramp filter cutoff at half the Nyquist frequency.

For each emission sinogram, 6 reconstructions were performed differing only in which attenuation map was used for the emission photon attenuation and scatter correction. These correspond to each of the 3 attenuation maps (no correction, correction for emission contamination only, and correction for emission contamination and scatter) and transmission sources $\left({ }^{68} \mathrm{Ge}\right.$ and ${ }^{57} \mathrm{Co}$ ). Before computing the attenuation correction factors from data acquired using the ${ }^{57} \mathrm{Co}$ transmission source (with a photon energy of approximately $122 \mathrm{keV}$ ), it was first necessary to convert the $\mu$-values for the annihilation photon energy (511 $\mathrm{keV}$ ). This conversion was done using a piecewise linear rescaling similar to the bilinear interpolation methods $(17,18)$ proposed for CT-based attenuation correction. All reconstructions were corrected for emission photon scatter using the single-scatter simulation method (11). Calibration to absolute quantitative reconstruction units was achieved by using a $0.3-\mathrm{MBq}{ }^{68} \mathrm{Ge}$ point source and rescaling the reconstructed counts (after all appropriate corrections) to a well-counter measurement.

\section{Possible Alternatives to Transmission Scatter Correction}

To illustrate the systematic errors that can be caused by rescaling or segmenting attenuation-map data without transmission scatter correction, we have reconstructed 2 sets of experimental emission data (using Fourier rebinning filtered backprojection with all the necessary corrections for quantitative PET described in the previous section) from the microPET Focus 120 . For the first experiment, we used a 30-mm-radius water cylinder filled with a uniform concentration of ${ }^{18} \mathrm{~F}$ activity with singles-mode transmission data acquired using a ${ }^{57} \mathrm{Co}$ source. For the second experiment, we used a 45-mmradius water cylinder also filled with a uniform concentration of ${ }^{18} \mathrm{~F}$ activity but with transmission data acquired using a ${ }^{68} \mathrm{Ge}$ source. These experiments were selected based on our previous work (2), which suggested that rescaling or segmentation may be problematic if the transmission data are not corrected for contamination from scattered photons. The cylinders investigated here are closer in size to a larger rat and may therefore be subject to more transmission scatter than occurs in a typical mouse study. The primary focus of these studies was to investigate the influence of transmission scatter and not emission contamination. Therefore, for both experiments, the transmission scans were performed after the ${ }^{18} \mathrm{~F}$ had decayed to negligible amounts (we estimate that, for both studies, less than $0.3 \%$ of the detected counts in the transmission data were due to emission contamination).

For the first experiment, we reconstructed the attenuation-map data with and without our scatter correction. For the attenuationmap data that had not been corrected for scatter, we also performed a slice-by-slice rescaling for the attenuation-map data so that the average reconstructed $\mu$-value inside the water cylinder was equal to the known $\mu$-value of water. For the second experiment, we reconstructed transmission data with and without our scatter correction using MAPtr (7), a reconstruction-based automated segmentation routine that is distributed as part of the microPET Focus software package. The MAPtr algorithm effectively segments the attenuation-map image using the a priori knowledge that the reconstructed image should contain only a limited number of materials and tissue types (each with unique linear attenuation coefficients).

\section{RESULTS}

\section{Primate Scanner Studies}

The analyses of the attenuation maps for the water cylinder and for the Macaca nemestrina monkey are shown in Figures 1 and 2, respectively, and described in the legends. The amounts of central-processing-unit (CPU) time required for the calculation of the scatter correction were between 17 and 26 min using a 2.2-GHz processor. 


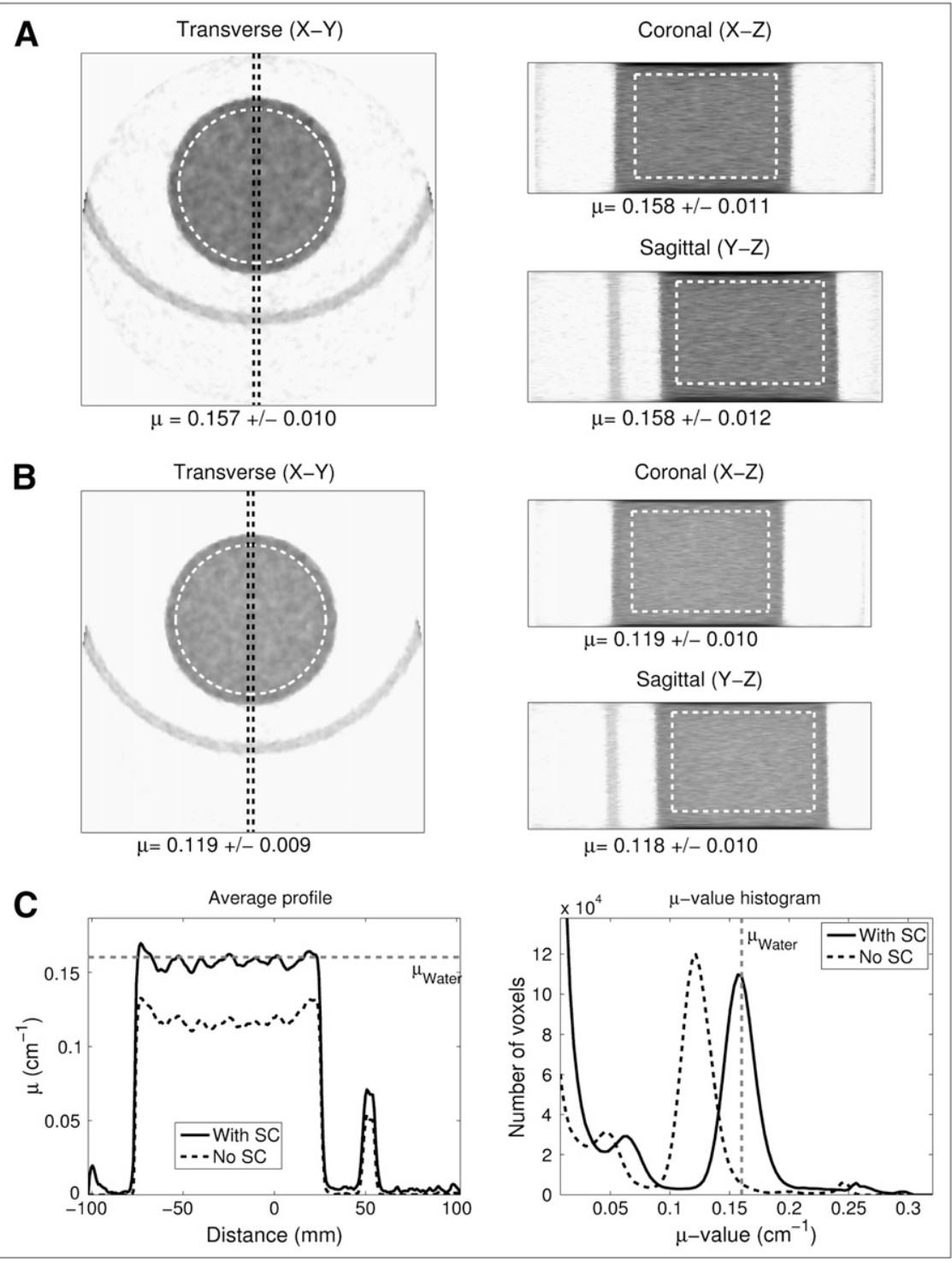

FIGURE 1. (A and B) Slices through reconstructed attenuation-map images with $(A)$ and without $(B)$ scatter correction. Results of ROI analysis in 3 perpendicular planes (transverse, coronal, and sagittal) for each attenuation map are shown. Average value and SD for all voxels within ROls (boundaries of which are indicated by dashed lines in images) are below each image. (C) Profiles through reconstructed data (averaged over 10 axial planes and over all data between 2 dashed black lines in transverse images) and histograms of reconstructed $\mu$-values for both attenuation maps. Data are for water cylinder (50-mm radius) and were acquired using microPET Focus 220 with ${ }^{57} \mathrm{Co}$ transmission source. In each plot, we also indicate expected true linear attenuation coefficient for water $\left(\mu_{\text {water }}=0.160 \mathrm{~cm}^{-1}\right.$ at $122 \mathrm{keV}(18)) . \mathrm{SC}=$ scatter correction.
Each ordered-subsets transmission reconstruction required about $21 \mathrm{~min}$ of CPU time using the same processor.

\section{Studies Using Postinjection Transmission Data}

Transmission Data Reconstruction. Figures 3 and 4 show examples of transverse slices through the reconstructed attenuation-map images for the cylinder and rodent studies, respectively. The images at top in each figure are reconstructions using the ${ }^{68} \mathrm{Ge}$ transmission data, and the images at bottom use the ${ }^{57} \mathrm{Co}$ data, which have been rescaled to the appropriate values for $511-\mathrm{keV}$ photons. In Supplemental Figures 1A and 1B (supplemental materials are available online only at http://jnm.snmjournals.org), we also show histograms of reconstructed $\mu$-values for the cylinder and rodent studies, respectively. For each study, histograms are shown for the ${ }^{68} \mathrm{Ge}$ and ${ }^{57} \mathrm{Co}$ attenuation maps and for the ${ }^{57} \mathrm{Co}$ attenuation maps that have been rescaled for $511-\mathrm{keV}$ photons. With a 2.2-GHz processor, the scatter correction for the cylinder and rodent studies required about 16 and 23 min of CPU time, respectively. Each ordered-subsets transmission reconstruction required about $3.5 \mathrm{~min}$ of CPU time using the same processor. This CPU time is about 10 times less than that required for reconstruction of the microPET Focus 220 data because of smaller sizes of the sinograms and images used for this scanner.

Emission Data Reconstruction. Supplemental Figure 2 shows transverse slices and profiles through the reconstructed activity-concentration image data for the cylinder and rodent studies. The activity image slices shown in Supplemental Figures 2A and 2B correspond to the same axial position as that shown for the attenuation-map images in Figures 3 and 4, respectively. For brevity, only the transverse images corresponding to the attenuation maps obtained using ${ }^{57} \mathrm{Co}$ transmission data with both types of correction are shown. The emission reconstructions and corrections required about 2 min of CPU time on the microPET Focus 
FIGURE 2. (A and B) Slices through reconstructed attenuation-map images with (A) and without (B) scatter correction. Results of ROI analysis in 3 perpendicular planes (transverse, coronal, and sagittal) for each attenuation map are shown. Average value and SD for all voxels within ROls (boundaries of which are indicated by dashed lines in images) are below each image. (C) Profiles through reconstructed data (averaged over 10 axial planes and over all data between 2 dashed black lines in transverse images) and histograms of reconstructed $\mu$-values for both attenuation maps. Data are for primate transmission data. For this study, profile data were averaged over only 3 axial planes and pass through skull, brain, sinus, and snout. Note improved contrast between tissue and sinus for attenuation maps with scatter correction relative to that with no scatter correction. We expect that correct $\mu$-value for soft tissue is approximately equal to $\mu_{\text {Water }}=0.160$ $\mathrm{cm}^{-1}$ (18). SC $=$ scatter correction.

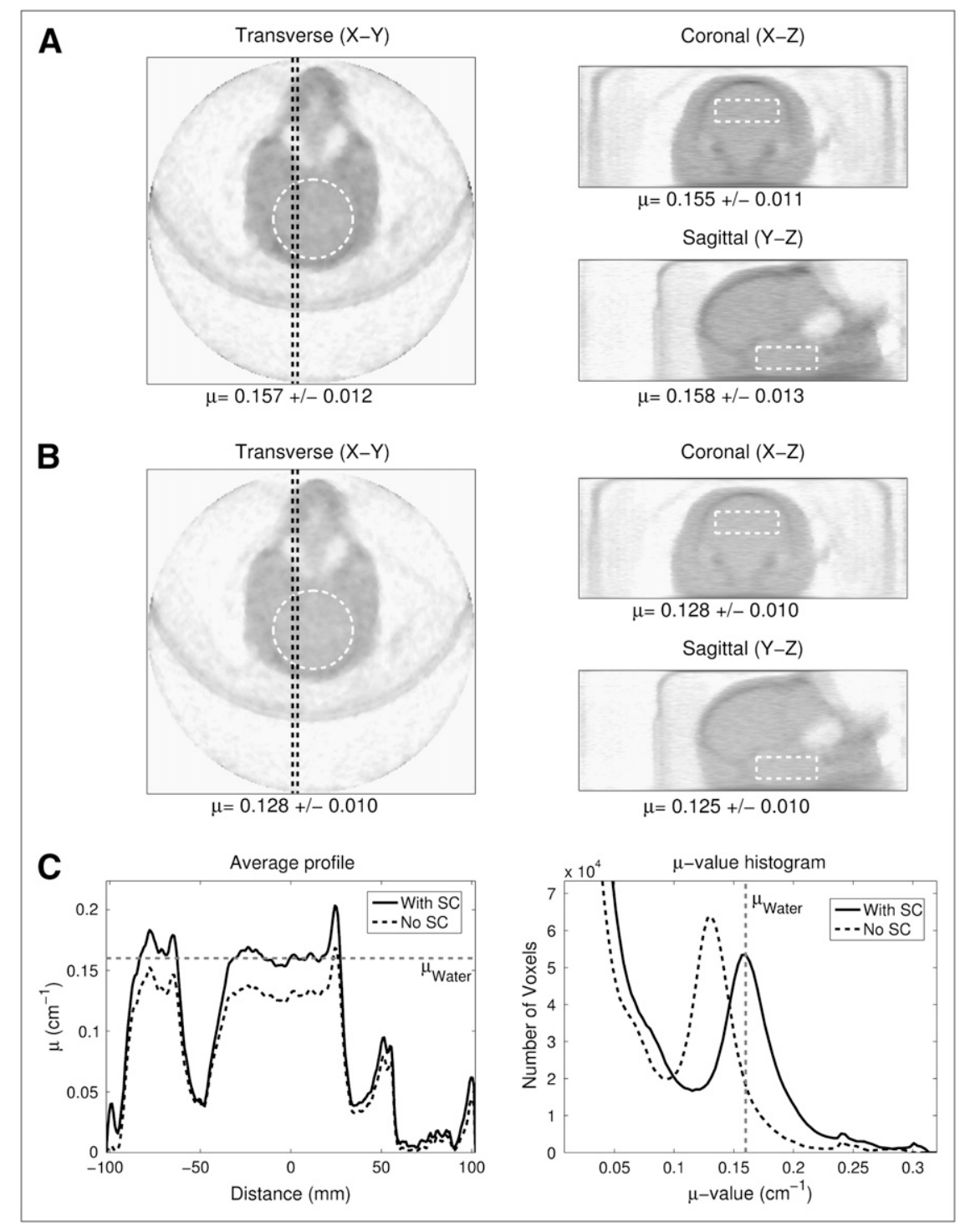

acquisition and processing computer. We converted the concentration images to units of total activity per voxel (by simply multiplying by the voxel volume) and summed over all transverse planes in which either the line source or the simulated tumor was visible. We then placed circular regions of interest (ROIs) (with radii of 4.3 and $7.8 \mathrm{~mm}$ for the linesource and tumor ROIs, respectively) on each summed image and determined the total amounts of activity within each ROI. Table 1 compares the total reconstructed activity within the ROIs with the measured activity estimated using a well counter.

\section{Possible Alternatives to Transmission Scatter Correction}

Attenuation-Map Rescaling. Figure 5A shows profiles through the attenuation-map data for the first water cylinder experiment (30-mm radius, ${ }^{57} \mathrm{Co}$ transmission source): scatter-corrected, uncorrected, and uncorrected and rescaled. Figure $5 \mathrm{~B}$ shows the corresponding data for the emission reconstructions that were corrected using these attenuation maps. In the profiles, we also indicate the attenuation coefficient for water $\left(\mu_{\text {water }}\right)$ and the average reconstructed emission activity using the scatter-corrected attenuation map $\left(\lambda_{\text {with SC}}\right)$. The location of the profiles is indicated in Supplemental Figure 3, which shows transverse attenuation and activity images for this experiment.

Attenuation-Map Segmentation. Figures 6A and 6B shows profiles through the segmented attenuation-map and reconstructed activity-concentration data, respectively, for the second water cylinder experiment $(45-\mathrm{mm}$ radius, ${ }^{68} \mathrm{Ge}$ transmission source). In the profiles, we also show the true attenuation coefficients for water and lung ( $\mu_{\text {water }}$ and $\mu_{\text {lung }}$, respectively) and the average activity ( $\left.\lambda_{\text {with }}{ }_{\text {sc }}\right)$ corresponding to the scatter-corrected attenuation map. Transverse attenuation and activity images for this study are shown in Supplemental Figures 4A and 4B, respectively. The attenuation-map data were reconstructed and 
A

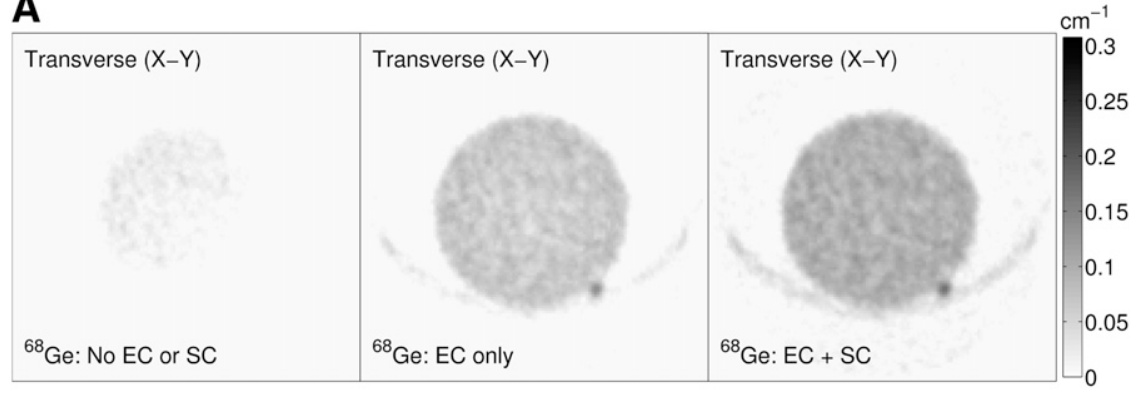

B

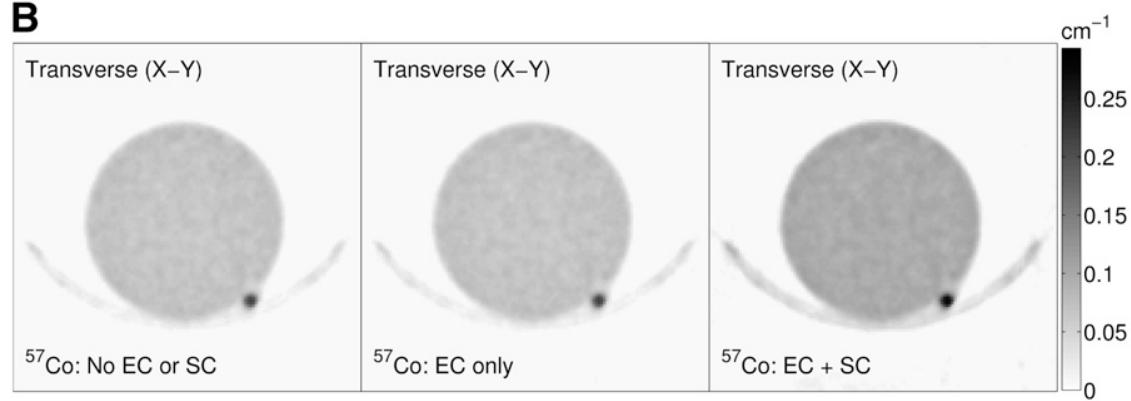

FIGURE 3. Comparison of transverse attenuation-map images (averaged over 3 axial planes) reconstructed using ${ }^{68} \mathrm{Ge}$ (A) and ${ }^{57} \mathrm{Co}(\mathrm{B})$ postinjection transmission data acquired on microPET Focus 120. Data are for line source attached to water cylinder and demonstrate influence of emission contamination correction (EC) and scatter correction (SC). segmented using transmission data with and without scatter correction.

\section{DISCUSSION}

\section{Primate Scanner Studies}

Figures 1 and 2 show that our reconstruction and scatter correction recovered linear attenuation coefficients ( $\mu$-values) within a few percentage points of their expected values for the singles-mode transmission data acquired using the microPET Focus 220. For example, with scatter correction the average reconstructed $\mu$-values for the ROI analysis shown on the attenuation-map images were within
$3 \%$ of their expected values for both the cylinder experiment and the primate study. The percentage errors in the reconstructed $\mu$-values without scatter correction varied for different sized objects (errors were between $19 \%$ and $22 \%$ for the primate study and were about $25 \%$ for the cylinder study) and also depend on the location of the ROI (for the primate study the errors become larger closer to the monkey's body, where there is more scatter from outside the field of view).

The profiles shown in Figures $1 \mathrm{C}$ and $2 \mathrm{C}$ indicate that our scatter correction reduces the radial variation due to scatter for the uniform cylinder. The profile for the primate

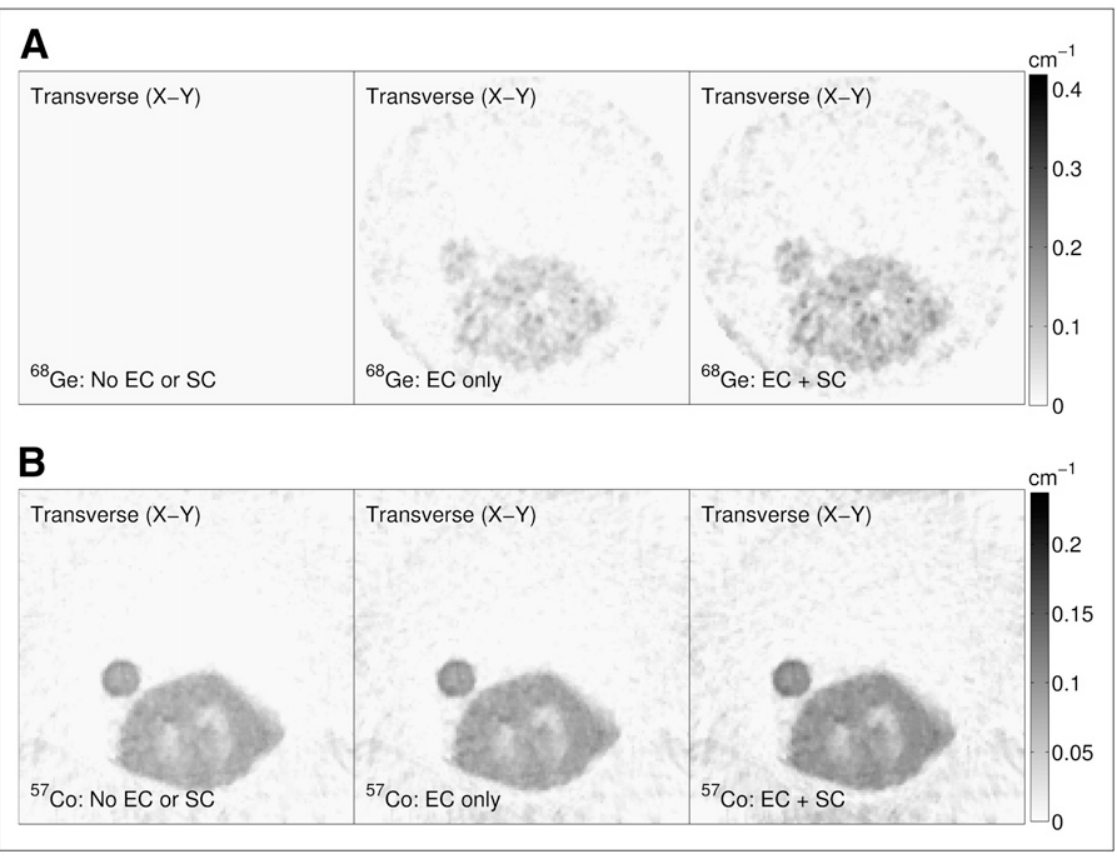

FIGURE 4. Comparison of transverse attenuation-map images (averaged over 3 axial planes) reconstructed using ${ }^{68} \mathrm{Ge}$ (A) and ${ }^{57} \mathrm{Co}(\mathrm{B})$ postinjection transmission data acquired on microPET Focus 120. Data are for postinjection rodent study. Images correspond to transverse slice of rat's torso and include parts of its lungs and circular cross section of external tumor on left sides of images. EC = emission-contamination correction; $\mathrm{SC}=$ scatter correction. 
TABLE 1

Comparison of Activity Within Circular ROls for Emission Images Reconstructed Using Attenuation Maps with Different Combinations of Corrections

\begin{tabular}{|c|c|c|c|c|}
\hline \multirow[b]{2}{*}{ Type of correction } & \multicolumn{2}{|c|}{$\begin{array}{l}\text { Cylinder-study } \\
\text { activity (MBq) }\end{array}$} & \multicolumn{2}{|c|}{$\begin{array}{l}\text { Rodent-study } \\
\text { activity (MBq) }\end{array}$} \\
\hline & ${ }^{68 \mathrm{Ge}}$ & ${ }^{57} \mathrm{Co}$ & ${ }^{68} \mathrm{Ge}$ & ${ }^{57} \mathrm{Co}$ \\
\hline $\begin{array}{l}\text { Both emission } \\
\text { contamination } \\
\text { and attenuation }\end{array}$ & 1.03 & 1.02 & 5.84 & 5.94 \\
\hline $\begin{array}{l}\text { Only emission } \\
\text { contamination }\end{array}$ & 0.84 & 0.88 & 5.20 & 5.53 \\
\hline None & 0.66 & 0.87 & 4.20 & 5.26 \\
\hline
\end{tabular}

Based on well-counter measurements, we estimate that approximately 0.9 and $6.0 \mathrm{MBq}$ of activity were present within ROls for cylinder and rodent studies, respectively.

data passes through several types of tissue, including the skull, the brain, a nasal sinus, and parts of the monkey's snout. For the soft-tissue regions of this profile (brain and parts of the snout), we obtain reconstructed $\mu$-values close to their expected values when scatter correction is applied. We also obtain better contrast (larger peak-to-valley ratios in the profiles) between reconstructed $\mu$-values for soft tissue and sinus (air) when our scatter correction is applied.

\section{Studies Using Postinjection Transmission Data}

Transmission Data Reconstruction. For the histograms shown in Supplemental Figures 1A and 1B, we expect to observe a peak corresponding to the reconstructed $\mu$-value of water (or, equivalently, soft-tissue). For the cylinder study using the ${ }^{68} \mathrm{Ge}$ transmission data and reconstructed with no emission-contamination or scatter correction, no discernible peak can be found in the histograms (Supplemental Fig. 1A), and the attenuation-map images (Fig. 3A) are clearly distorted because of the presence of the line source (on the lower right side of the cylinder). This situation was even worse for the rodent study, in which the $\mu$-value for almost every voxel of the ${ }^{68} \mathrm{Ge}$ attenuation map with no correction for emission contamination or scatter was equal to zero (Fig. $4 \mathrm{~A})$. This finding implies that for most lines of response the number of counts was greater in the ${ }^{68} \mathrm{Ge}$ transmission data than in the blank data (which would give physically incorrect negative $\mu$-values if the nonnegativity constraint were not enforced during reconstruction). On the basis of our mockscan measurements, we estimate that the emission contamination contributed $19 \%$ and $78 \%$ of the counts in the ${ }^{68} \mathrm{Ge}$ transmission data for the cylinder and rodent studies, respectively. These results indicate how important emission contamination correction is for ${ }^{68} \mathrm{Ge}$ singles-mode transmission data.

For the ${ }^{57} \mathrm{Co}$ transmission data, we estimate that emission contamination contributed only $0.6 \%$ and $5.7 \%$ of the total counts for the cylinder and rodent transmission scans, respectively. It is clear that the contribution from emission photons depends on the amount of emission activity relative to the transmission source, the energy of the transmission source, and the energy discrimination settings used. Emission contamination can be reduced significantly, relative to
FIGURE 5. Profiles through transverse attenuation images (A) and activityconcentration images (B) for 30 -mm-radius water cylinder using ${ }^{57} \mathrm{Co}$ transmission data. Profiles shown pass through center of cylinder (Supplemental Fig. 3) and were averaged over all axial planes to reduce noise and illustrate systematic effects of scatter in transmission data. Data correspond to scatter-corrected, uncorrected, and rescaled uncorrected attenuation maps. SC = scatter correction.

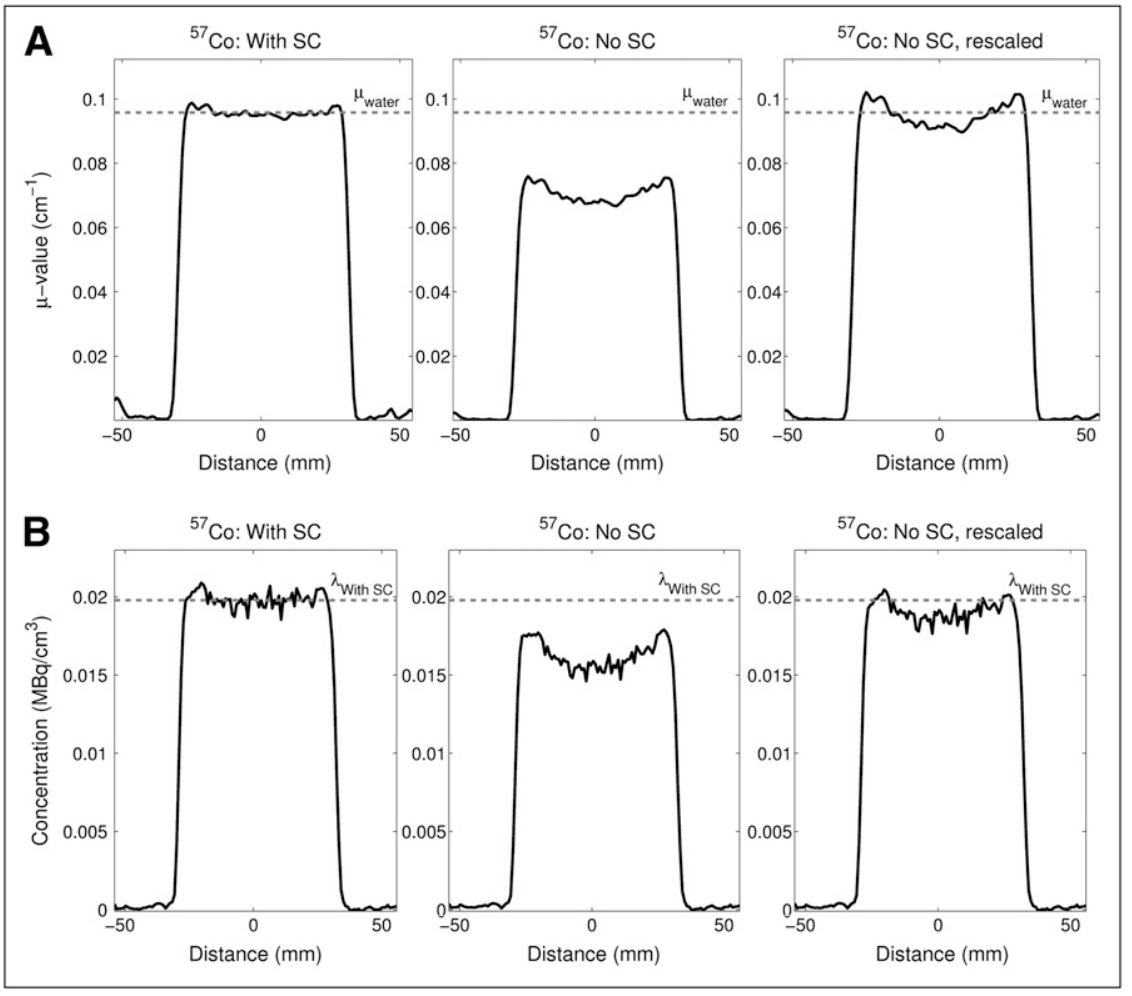




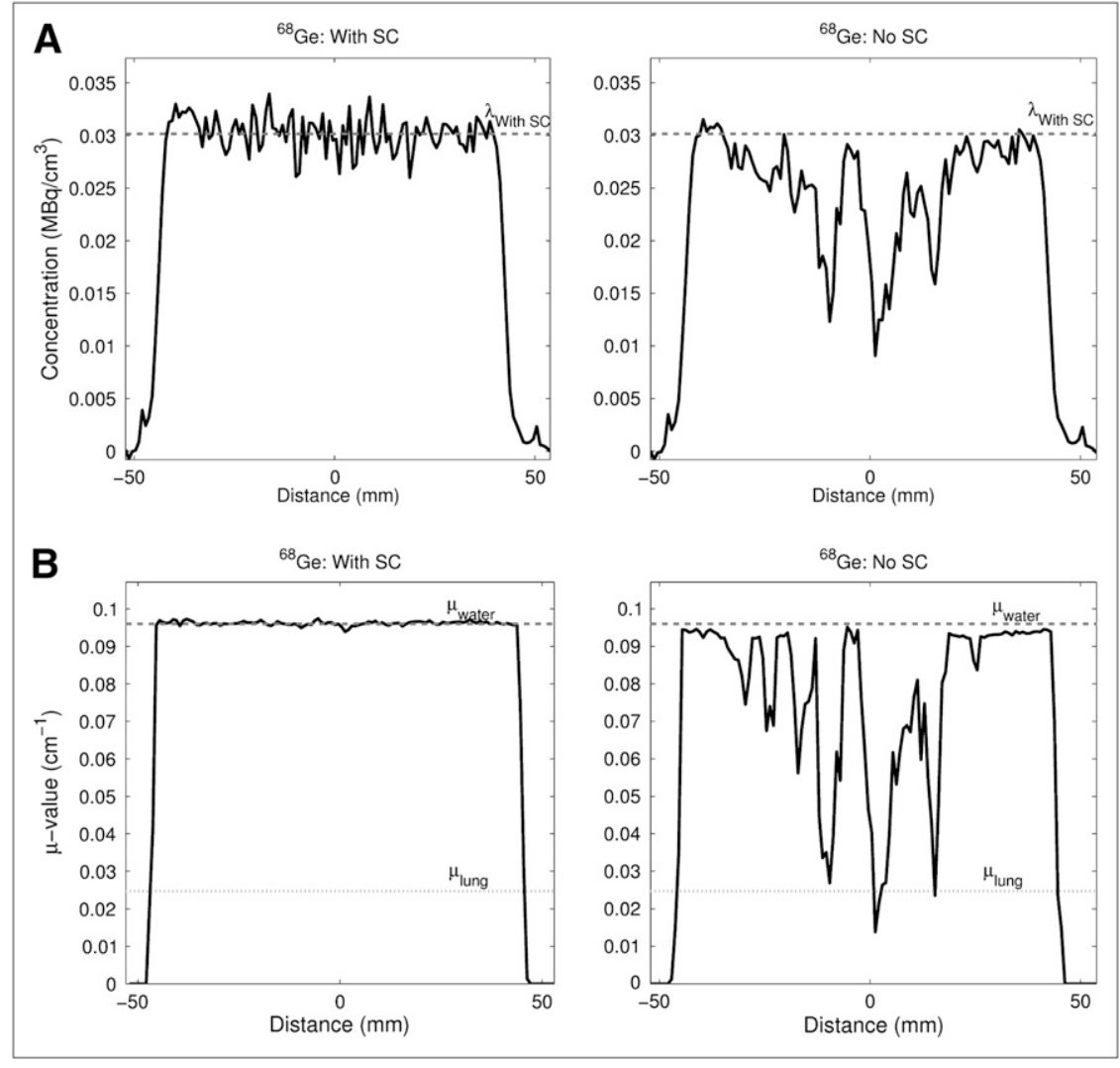

FIGURE 6. Profiles through transverse attenuation images (A) and activity-concentration images $(B)$ for central slice of $45-\mathrm{mm}$-radius water cylinder using ${ }^{68} \mathrm{Ge}$ transmission data. Profiles pass through center of cylinder (Supplemental Fig. 4) and were averaged over 10 axial planes to reduce noise in emission data. Transmission data were reconstructed using MAPtr, automated reconstruction-based segmentation method of Nuyts et al. (7), with and without transmission scatter correction. SC $=$ scatter correction. that in ${ }^{68} \mathrm{Ge}$ transmission data, simply by using energy discrimination with a lower-energy $\gamma$-emitting transmission source such as ${ }^{57} \mathrm{Co}$.

Our results indicate that it is not sufficient to perform only the emission-contamination correction and that, to obtain accurate $\mu$-values, one must also apply scatter correction. When correction was applied only for emission contamination in the cylinder study, the $\mu$-value histogram peak positions in Supplemental Figure 1A were 36\% and 30\% lower than the expected values for water using the ${ }^{68} \mathrm{Ge}$ and ${ }^{57} \mathrm{Co}$ transmission data, respectively. For the attenuation maps with both corrections, however, the histogram peak positions were within $2 \%$ of their expected values for water for the cylinder study using each transmission source.

For the rodent study using the ${ }^{57}$ Co transmission source, the peaks in the $\mu$-value histograms in Supplemental Figure $1 \mathrm{~B}$ were $25 \%$ (no emission contamination correction) and $16 \%$ (emission contamination correction only) lower than the expected value for soft tissue when transmission scatter correction was not applied. When both corrections were applied, the histogram peak value was within $1 \%$ of its expected value for the ${ }^{57} \mathrm{Co}$ transmission data. The attenuation maps with emission contamination corrections (emission contamination correction only and both corrections) for the rodent study using the ${ }^{68} \mathrm{Ge}$ transmission data were extremely noisy and no discernible peaks could be found in either of their $\mu$-value histograms. We performed an ROI analysis (data not shown) in 3 perpendicular planes (transverse, coronal, and sagittal) for the attenuation maps recon- structed from the ${ }^{68} \mathrm{Ge}$ transmission data using emission contamination correction only and both corrections. We found that, despite their poor image quality, the images reconstructed using both corrections had average $\mu$-values within $\pm 13 \%$ of their expected values for soft-tissue ROIs. The average values, for the same ROIs placed on the images corrected for emission contamination only, were between $30 \%$ and $34 \%$ lower than the expected value.

For the postinjection transmission data analyzed here, the ${ }^{68} \mathrm{Ge}$ and ${ }^{57} \mathrm{Co}$ blank data (which had the same duration as the transmission data) happened to have approximately the same average number of counts (about 350 per bin) in each bin of the single-slice rebinned sinogram data. This finding suggests that the improved quality of the ${ }^{57} \mathrm{Co}$ images is largely due to the lower amounts of emission contamination relative to that for the ${ }^{68} \mathrm{Ge}$ data and the better contrast between $\mu$-values at lower energy.

The piecewise linear rescaling we have used here correctly converts the linear attenuation coefficients for water from the values expected for $122-\mathrm{keV}$ photons to those for $511-\mathrm{keV}$ photons. For the histograms shown in Supplemental Figures $1 \mathrm{~A}$ and $1 \mathrm{~B}$, the water peaks are correctly shifted to the appropriate $\mu$-values for the attenuation maps with both types of corrections. Based on values given by Berger et al. (19), we estimate that the effective linear attenuation coefficient for the line-source material is approximately $0.27 \mathrm{~cm}^{-1}$ at $511 \mathrm{keV}$. We placed ROIs inside the vicinity of the line source for the ${ }^{57} \mathrm{Co}$ attenuation map (applying both corrections and rescaling for $511-\mathrm{keV}$ 
photons) and found that the average reconstructed $\mu$-values were between 0.25 and $0.28 \mathrm{~cm}^{-1}$.

Emission Data Reconstruction. The profiles shown in Supplemental Figures 2C and 2D indicate that the reconstructed activity concentrations are strongly influenced by the correction applied during the attenuation-map reconstructions. If we examine the results of the ROI analysis summarized in Table 1, we find that the total activities for the emission images reconstructed using only emissioncontamination correction were between $7 \%$ and $18 \%$ lower than the corresponding values for the images with both corrections. The total reconstructed activities for the images with no correction were between $35 \%$ and $15 \%$ lower than those for the images with both corrections. We also found that the total activity within the ROIs for each study were consistent (agreed to within 2\%) for images reconstructed using either ${ }^{68} \mathrm{Ge}$ or ${ }^{57} \mathrm{Co}$, provided that both emissioncontamination and scatter corrections were applied.

If we compare the total reconstructed activities in Table 1 with the total activities estimated from the well-counter measurements, we find that values for the images with both corrections were within about $3 \%$ of the measured values for the rodent study but were overestimated by about $14 \%$ for the line-source and cylinder data. However, the well counter measurement for the pointlike tumor used for the rodent study is subject to less uncertainty than that of the line source. There are systematic errors associated with measuring the activity of an extended line source using a well counter, because it is calibrated for point sources centered in the bottom of the well. For example, we have observed that the measured activity for a pointlike source can be as much as $6 \%$ lower when the source is located 30$40 \mathrm{~mm}$ above the bottom of the well counter, compared with when it is centered in the bottom of the well. Because the line source is $115 \mathrm{~mm}$ long, the well-counter measurement for its activity may also be underestimated. Another source of uncertainty occurs because the well-counter measurement for the line source is subject to photon attenuation, whereas the reconstructed image has been corrected for attenuation. In the activity values given in the legend of Table 1, we have compensated for this effect by multiplying the well-counter measured activity by an approximate attenuation correction factor. We estimate that $9 \%$ of photons are attenuated in the line-source material, assuming that all photons are emitted from the center of the line source in a plane perpendicular to the length of the line source. It is possible that we have underestimated the attenuation effect, because photons emitted from the center of the line source in any nonperpendicular plane will be subject to more photon attenuation.

\section{Possible Alternatives to Transmission Scatter Correction}

Figures 5 and 6 and Supplemental Figures 3 and 4 show that the erroneous radial variation, which is visible in attenuation-map data that have not been corrected for scatter, causes systematic errors in the reconstructed emission images. Figure 5 indicates that rescaling the noncorrected attenuation maps does not eliminate the radial variation and that these errors propagate into the reconstructed emission images. The most uniform activityconcentration profiles were obtained using our scatter correction method. Figure 6 indicates that this radial variation due to scatter in the attenuation-map images also can lead to errors in automated segmentation routines. In the uncorrected attenuation-map profiles and images shown in Figure 6A and Supplemental Figure 4A, many voxels are incorrectly assigned the linear attenuation coefficient for lung tissue. As we can see from the activity profiles and images shown in Figure 6B and Supplemental Figure 4B, this type of segmentation error causes an underestimation of the reconstructed activity for regions lying deeper inside the object (especially for regions incorrectly assigned the $\mu$-value for lung) relative to those closer to the surface. This is not meant to be a criticism of the segmentation method of Nuyts et al. (7) but is instead an indication of the importance of accurate scatter correction for singles-mode transmission data. When our scatter correction is applied, these segmentation errors do not occur (all voxels are assigned the correct $\mu$-value for water).

We have found in the current and our previous work (2) that errors in the reconstructed $\mu$-values without scatter correction can vary widely depending on the size of the subject, on the transmission source, and on the radial and axial positions within each attenuation-map image. Transmission scatter is most significant for subjects that are large relative to the PET scanner ring diameter (primates or heavier rats) and for regions of highly nonuniform attenuation (torso or whole-body imaging). Even for small animals such as mice, however, we have found that transmission scatter correction can be important (e.g., without scatter correction the reconstructed attenuation coefficients for a mouse were $19 \%$ lower than the expected value for soft tissue (2)). These results suggest that, for animal studies, the errors that could be caused by using rescaled or segmented attenuation maps without scatter correction may be difficult to predict and could result in misleading reconstructed emission images and errors in the biologic parameters derived from them. For example, the measurement of neuroreceptor binding potentials in small-animal PET can be particularly sensitive to attenuation errors, because this measurement depends critically on the relative amounts of striatal and cerebellar activity (20). In rats, the cerebellum is in a region subject to higher attenuation (and, as a result, higher transmission photon scattering) than is the striatum. The rescaling and segmentation errors for attenuation maps without scatter correction, as illustrated in Figures 5 and 6 and Supplemental Figures 3 and 4 , would lead to errors in this type of activity ratio.

\section{CONCLUSION}

Our results indicate that our reconstruction and scatter correction procedure can easily be adapted to different PET 
scanners (primate and rodent models of the microPET Focus) and different imaging protocols (pre- and postinjection data acquisition). For all cases, the average reconstructed $\mu$-values with scatter correction were within $3 \%$ of the expected values for water and soft tissue for both phantom and animal studies. Without scatter correction (and emission contamination correction for postinjection transmission), the reconstructed $\mu$-values were biased and varied widely depending on the size of the object, the local position within the object, the amount of emission activity within the scanner, and the type of transmission source. For the postinjection transmission data acquired using the microPET Focus 120, we found that the accuracy of reconstructed emission activity concentrations depends strongly on the scatter and emission-contamination correction applied to the singles-mode transmission data. We also found that our scatter correction algorithm is more accurate than the existing alternatives (segmentation or rescaling of uncorrected attenuation maps).

\section{ACKNOWLEDGMENTS}

We thank Dr. Tom Videen and Dr. Joel Perlmutter for providing the microPET Focus 220 data. We also thank Siobhan McCormick and Rick Kornelsen for their assistance with animal experiments. This research was supported by the Natural Sciences and Engineering Research Council of Canada.

\section{REFERENCES}

1. Smith R, Karp J, Muehllehner G, Gualtieri E, Benard F. Singles transmission scans performed post-injection for quantitative whole body PET imaging. IEEE Trans Nucl Sci. 1997;44:1329-1335.

2. Vandervoort E, Sossi V. An analytical scatter correction for singles-mode transmission data in PET. IEEE Trans Med Imaging. 2008;27:402-412.
3. Laforest R, Longford D, Siegel S, Newport DF, Yap J. Performance evaluation of the microPET ${ }^{\circledR}$-FOCUS-F120. IEEE Trans Nucl Sci. 2007;54:42-49.

4. Kim JS, Lee JS, Im KC, et al. Performance measurement of the microPET focus 120 scanner. J Nucl Med. 2007;48:1527-1535.

5. Tai Y, Ruangma A, Rowland D, et al. Performance evaluation of the microPET Focus: a third generation microPET scanner dedicated to animal imaging. $\mathrm{J} \mathrm{Nucl}$ Med. 2005;46:455-463.

6. Zaidi H, Diaz-Gomez M, Boudraa A, Slosman D. Fuzzy clustering-based segmented attenuation correction in whole-body PET imaging. Phys Med Biol. 2002;47:1143-1160.

7. Nuyts J, Dupont P, Stroobants S, Maes A, Mortelmans L, Suetens P. Evaluation of maximum-likelihood based attenuation correction in positron emission tomography. IEEE Trans Nucl Sci. 1999;46:1136-1141.

8. Daube-Witherspoon ME, Muehllehner G. Treatment of axial data in threedimensional PET. J Nucl Med. 1987;28:1717-1724.

9. Erdogan H, Fessler J. Ordered subsets algorithms for transmission tomography. Phys Med Biol. 1999;44:2835-2851.

10. Huber P. Robust Statistics, New York, NY: Wiley; 1981.

11. Watson CC. New, faster, image-based scatter correction for 3D PET. IEEE Trans Nucl Sci. 2000;47:1587-1594.

12. Vandervoort E, Camborde ML, Jan S, Sossi V. Monte Carlo modelling of singlesmode transmission data for small animal PET scanners. Phys Med Biol. 2007;52:3169-3184.

13. Dandekar M, Tseng J, Gambhir S. Reproducibility of ${ }^{18}$ F-FDG microPET studies in mouse tumor xenografts. J Nucl Med. 2007;48:602-607.

14. Humm JL, Lee J, O'Donoghue J, et al. Changes in FDG tumor uptake during and after fractionated radiation therapy in a rodent tumor xenograft: the effects of stereotactic radiotherapy. Clin Positron Imaging. 1999;2:289-296.

15. Badawi RD, Miller M, Bailey DL, Marsden P. Developments in componentbased normalization for 3D PET. Phys Med Biol. 1999;44:571-594.

16. Defrise M, Kinahan P, Townsend D, Michel C, Sibomana M, Newport D. Exact and approximate rebinning algorithms for 3-D PET data. IEEE Trans Med Imaging. 1997;16:145-158.

17. Burger C, Goerres G, Schoenes S, Buck A, Lonn A, von Schulthess G. PET attenuation coefficients from CT images: experimental evaluation of the transformation of CT into PET 511-keV attenuation coefficients. Eur J Nucl Med Mol Imaging. 2002;29:922-927.

18. Bai C, Shao L, Silva AD, Zuo Z. A generalized model for the conversion from CT numbers to linear attenuation coefficients. IEEE Trans Nucl Sci. 2003;50: $1510-1515$.

19. Berger M, Hubbell J, Seltzer S, et al. XCOM: photon cross section database. Gaithersburg, MD: National Institute of Standards and Technology; 2006. Available at: http://physics.nist.gov/xcom. Accessed August 22, 2008

20. Hume SP, Meyers R, Bloomfield P, et al. Quantitation of carbon-11-labeled raclopride in rat striatum using positron emission tomography. Synapse. 1992;12:47-54. 